\title{
Norm-Minimized Scattering Data from Intensity Spectra
}

\author{
Alexander Seel, ${ }^{1}$ Arman Davtyan, ${ }^{2}$ Ullrich Pietsch, ${ }^{2}$ and Otmar Loffeld ${ }^{1}$ \\ ${ }^{1}$ Center for Sensor Systems, University of Siegen, 57068 Siegen, Germany \\ ${ }^{2}$ Faculty of Science and Engineering, University of Siegen, 57068 Siegen, Germany
}

Correspondence should be addressed to Alexander Seel; alexander.seel@uni-siegen.de

Received 16 February 2016; Accepted 20 April 2016

Academic Editor: Srdjan Stankovic

Copyright (C) 2016 Alexander Seel et al. This is an open access article distributed under the Creative Commons Attribution License, which permits unrestricted use, distribution, and reproduction in any medium, provided the original work is properly cited.

We apply the $\ell_{1}$ minimizing technique of compressive sensing (CS) to nonlinear quadratic observations. For the example of coherent $\mathrm{X}$-ray scattering we provide the formulas for a Kalman filter approach to quadratic CS and show how to reconstruct the scattering data from their spatial intensity distribution.

\section{Introduction}

The rapidly growing Si technology in semiconductor electronics $[1,2]$ opens the possibility to grow III-V inorganic nanowires, such as GaAs, InAs, or InP, which were supposed to have potential $[3,4]$ to become building blocks in a variety of nanowire-based nanoelectronic devices, for example, in nanolaser sources [5] or nanoelectronics [6]. Such epitaxially grown nanowires are repeating the crystal orientation of the substrate and usually grow in Wurtzite (WZ) or ZincBlende $(\mathrm{ZB})$ structure differing in the stacking sequences $A B A B A B$ and $A B C A B C A B C$, respectively, of the atomic bilayers. Theoretical predictions on the electronic properties [7] of these nanowires show that stacking sequences with WZ and ZB segments considerably differ in the conductivity. However, during the nanowire growth stacking faults, the mixing of $\mathrm{ZB}$ and $\mathrm{WZ}$ segments takes place, and twin defects [8] appear. As these defects have their own impact on the conductivity and band structure there is great interest in knowing the exact stacking sequence which can be studied by, for example, Transmission Electron Microscopy [9]. But, as this is a destructive method, it is impossible to use the nanowire after the structural studies. Nowadays the 3rd generation synchrotron sources and rapidly developing focusing devices like Fresnel Zone Plates opens new fields of nondestructive Xray imaging. For example, in the Coherent X-ray Diffraction Imaging (CXDI) experiments, an isolated nanoobject is illuminated with coherent $\mathrm{X}$-ray radiation and the scattered intensity is measured by a $2 \mathrm{D}$ detector $[10,11]$ under the Bragg condition. The diffraction patterns are structure-specific and encode the information about the electron density of the sample and thus the stacking sequence of the atomic bilayers formally by Fourier transform. However, because of sensor physics, the phase information is lost in CXDI measurement since the measured intensity pattern is the modulus square of the scattered X-rays and no inverse Fourier transform can be directly applied to recover the stacking sequence. The classical approach with the Patterson function [12] fails as the number of expected randomly distributed stacking faults is too high. Other inversion algorithms $[13,14]$ could be used instead to reconstruct the lost phase information: although dual space iterative algorithms [15] have been shown to converge under specific conditions [16] there still remain some convergence problems for a number of cases when not enough preliminary information regarding the structure of the object is previously known [17]. Indeed ptychography type of experiments $[18,19]$ were suggested to determine at least the relative phases of the bilayers by interfering adjacent Bragg reflections. But this type of experiments is more difficult to realize, as it requires higher stability in comparison to conventional CXDI and the longer measurement time can, however, influence the sample [20].

Due to the principal loss in phase information the scattering data can be considered to be undersampled and algorithms tailored to this lack of information, like basis pursuit approaches [21] realized by minimizing the $\ell_{1}$ norm of 
sparse vectors in an appropriate basis or phase retrieval via matrix completion [22-24], could be tested for reconstruction from conventional CXDI measurements: as these data are recorded without structured illumination for applying matrix completion we focus on utilizing the $\ell_{1}$ minimizing technique to reconstruct undersampled sparse signals [25, 26] as vectors from linear mappings between the signal and the observations. For an overview of this so-called compressive sensing (CS) see the textbook [27]. The method of CS aims at a signal's sparse support which can be estimated by, for example, Kalman filtering [28]. As the underlying filter formulas also relate observations and signals to be reconstructed by linear mappings this technique meets CS and was also used to explicitly minimize the $\ell_{1}$ norm by so-called pseudomeasurements [29]. For an example see the reconstruction from a random sample of Fourier coefficients [30], where the dimensionality of the underlying Kalman gain matrices can be reduced utilizing the null space of the sensing matrix [31]. Even for nonlinear mappings of signals Kalman filtering applies by using Jacobians rather than constant sensing matrices. These extended Kalman filters (EKF) are used for, for example, tracking issues [32] or robotics [33] and matching the sensing problem of the quadratic nonlinearities in the spatial intensity distribution of CXDI.

The paper is organized as follows. In Section 2 we set up the observation model for modulus-squared amplitudes of intensity distributions in coherent X-ray scattering and point out the relation to the $\ell_{1}$ minimization. In Section 3 we give a brief overview of the linear Kalman filter model and show how to incorporate the complex nonanalytic $\ell_{1}$ norm as a linearized observation to meet the minimizing strategy of CS. In this framework we prove a convergence concept for the $\ell_{1}$ minimization in the reconstruction scheme. In Section 4 we apply our findings to $1 \mathrm{D}$ and 2D Fourier data and remark in Section 5 on future considerations.

\section{Motivation}

2.1. Intensity Spectra in Coherent Scattering Experiments. Exposing crystals to nondestructive coherent $\mathrm{X}$-ray radiation in $d$ dimensions the amplitude of the elastically scattered radiation is proportional to the Fourier transform [34]

$$
S(\mathbf{q})=\int \mathrm{d}^{d} x \mathrm{e}^{\mathrm{i}\langle\mathbf{q} \mid \mathbf{r}\rangle} b(\mathbf{r})
$$

of the electron density $b(\mathbf{r})$, where the vector $\mathbf{q}:=\mathbf{k}_{\text {out }}-$ $\mathbf{k}_{\text {in }}$ is a parametrization of the direction where radiation is detected and (multiplied by Planck's constant) also describes the momentum transfer in a kinematical scattering theory; $\mathbf{k}_{\text {in }}$ is the incident direction whereas $\mathbf{k}_{\text {out }}$ represents the outgoing direction for radiation with wave vectors $\left\|\mathbf{k}_{\text {out }}\right\|=$ $\left\|\mathbf{k}_{\text {in }}\right\|=2 \pi / \lambda$ of wavelength $\lambda$ (see Figure 1 ).

Following standard textbooks, for example, [34], one deals with two sets of basis vectors to characterize the scattering. The spatial vectors are represented in the basis $\left\{\mathbf{a}_{1}, \ldots, \mathbf{a}_{d}\right\}$ of the grid whereas the reciprocal basis

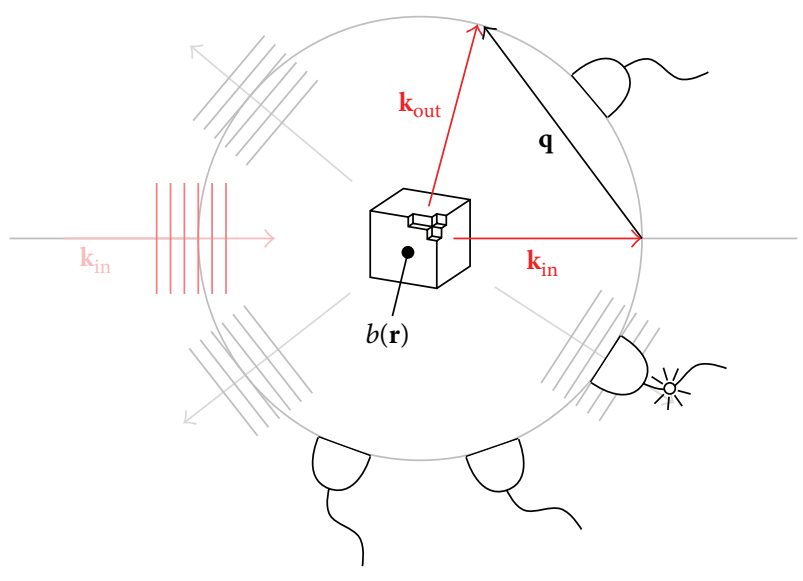

Figure 1

$\left\{\mathbf{b}_{1}, \ldots, \mathbf{b}_{d}\right\}$ is used for wave vectors $\mathbf{q}, \mathbf{k}_{\text {in }}, \mathbf{k}_{\text {out }}$ relying on the normalization

$$
\left\langle\mathbf{a}_{j} \mid \mathbf{b}_{k}\right\rangle=2 \pi \delta_{j k}, \quad j, k=1, \ldots, d .
$$

As the units of lengths and wave vectors are carried by the basis sets the scalar products read, with dimensionless factors $y_{j}$ and $\kappa_{k}$,

$$
\begin{gathered}
\mathbf{r}=\sum_{j=1}^{d} y_{j} \mathbf{a}_{j}, \\
\mathbf{q}=\sum_{k=1}^{d} \kappa_{k} \mathbf{b}_{k}, \\
\langle\mathbf{q} \mid \mathbf{r}\rangle=2 \pi \sum_{j=1}^{d} y_{j} \kappa_{j} .
\end{gathered}
$$

Restricting to a finite grid with $n_{1}, n_{2}, \ldots, n_{d}$ lattice sites in each direction with periodic boundary conditions the possible grid positions and wave vectors allowing for a discrete Fourier transform ${ }^{1}$ read, for all $j=1,2, \ldots, d$,

$$
\begin{aligned}
& y_{j} \in\left\{0,1, \ldots, n_{j}-1\right\}, \\
& \kappa_{j} \in\left\{\frac{0}{n_{j}}, \frac{1}{n_{j}}, \ldots, \frac{n_{j}-1}{n_{j}}\right\} .
\end{aligned}
$$

Thus the scalar product separates into the $d$ spatial dimensions according to

$$
\langle\mathbf{q} \mid \mathbf{r}\rangle=\sum_{j=1}^{d} \frac{2 \pi k_{j} r_{j}}{n_{j}}, \quad k_{j}, r_{j} \in\left\{0,1, \ldots, n_{j}-1\right\},
$$

where $k_{j}$ are related to the lattice positions in direction of the lattice constant $\mathbf{a}_{j}$ and $r_{j}$ refer to a discretized wave vector in direction of the corresponding reciprocal basis $\mathbf{b}_{j}$. For example, a $d=2$-dimensional regular hexagonal lattice $\left\{\mathbf{a}_{1}, \mathbf{a}_{2}\right\}$ encloses $120^{\circ}$ angles (see Figure 2 ). 

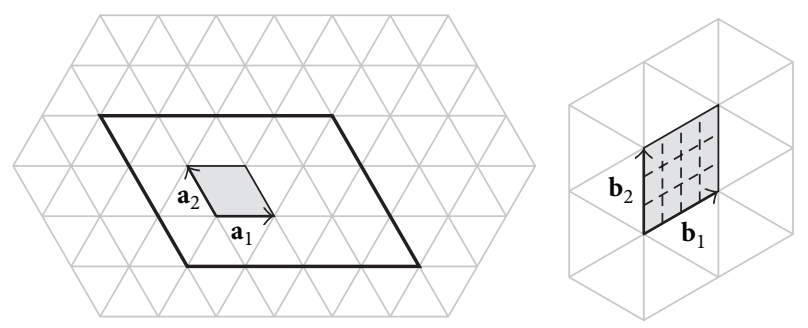

Figure 2

The bold parallelogram represents periodic boundary conditions with, for example, $n_{1}=4$ and $n_{2}=3$ fragmenting the 1st Brillouin zone, the elementary cell spanned by $\left\{\mathbf{b}_{1}, \mathbf{b}_{2}\right\}$ in reciprocal space, into a subgrid according to (4). With the normalization (2) the reciprocal lattice encloses angles of $60^{\circ}$ with a fixed orientation with respect to $\left\{\mathbf{a}_{1}, \mathbf{a}_{2}\right\}$.

2.2. Setting. Of course for the application the formulas are only needed up to $d=3$ dimensions: nanowires can be characterized by the stacking sequence of atomic bilayers which are shifted laterally and vertically with respect to each other. In coherent $\mathrm{X}$-ray diffraction summing up all scattered radiation from a certain bilayer perpendicular to the growth direction $\mathbf{a}_{3}$ yields a complex scattering amplitude $x_{k} \in \mathbb{C}$ associated with the $k$ th bilayer spanned by $\left\{\mathbf{a}_{1}, \mathbf{a}_{2}\right\}$. Due to the hexagonal lattice and with respect to an arbitrary reference bilayer there are three different phase factors:

$$
\left\{1, \exp \left(\frac{2 \pi \mathrm{i}}{3}\left(2 \kappa_{1}+\kappa_{2}\right)\right), \exp \left(\frac{2 \pi \mathrm{i}}{3}\left(\kappa_{1}+2 \kappa_{2}\right)\right)\right\}
$$

for the amplitudes left $[34,35]$. Wave vectors $\mathbf{q}$ sensitive to the arrangement of the bilayers are selected from directions related to the Bragg condition by $\kappa_{1}-\kappa_{2} \neq 3 N$ with $N, \kappa_{1}, \kappa_{2} \in \mathbb{Z}$ yielding $\{1, \exp (-2 \pi \mathrm{i} / 3), \exp (2 \pi \mathrm{i} / 3)\}$, which directly relates to the three possible lateral shifts. With respect to the experimental setup [17] this is satisfied by $\kappa_{1}=0$ and $\kappa_{2}=1$. Recovering the stacking sequence of these relative phase factors by varying $\kappa_{3}$ directly reveals the Zinc-Blende or Wurtzite structure of the wire along with their stacking faults.

Carrying out the remaining summation over all equidistant bilayers in growth direction $\mathbf{a}_{3}$ mathematically corresponds to the 1D discrete Fourier transform

$$
S_{r}=\sum_{k=0}^{n-1} \mathrm{e}^{2 \pi \mathrm{i} k r / n} x_{k}, \quad r=0,1, \ldots, n-1
$$

of the periodically continued complex scattering amplitudes $x_{k}$ combined with the vector $\mathbf{x}$. Each nonzero entry represents one bilayer and $r$ refers to the discretized component $\kappa_{3}$ of the q vector in the reciprocal basis corresponding to the growth direction. As the outcome of the detector is the measured intensity rather than the amplitude the observation is the squared signal

$$
\left|S_{r}\right|^{2}=\left\langle\mathbf{x} \| T_{r} \mid \mathbf{x}\right\rangle, \quad r=0,1, \ldots, n-1,
$$

where the Fourier coefficients build a Hermitian Toeplitz matrix

$$
T_{r}:=\left(\mathrm{e}^{-(2 \pi \mathrm{i} r / n)(p-q)}\right)_{p q} \in \mathbb{C}^{n \times n}, \quad r=0,1, \ldots, n-1,
$$

showing orthogonality $T_{r} T_{s}=n \delta_{r s} T_{r}$ with respect to multiplication and a completeness property $T_{0}+T_{1}+\cdots+T_{n-1}=n \mathbb{1}_{n}$ with respect to summation. Clearly, due to the squaring, the signal (8) is invariant under both the transformation $\mathbf{x} \rightarrow$ $\mathrm{e}^{\mathrm{i} \phi} \mathbf{X}$ with any global phase $\phi \in \mathbb{R}$ and the reflection $x_{k} \rightarrow$ $\bar{x}_{n-1-k}, k=0, \ldots, n-1$, on the lattice. As the spatial directions exhibit periodic boundary conditions the signal also remains invariant under discrete translations $x_{k} \rightarrow x_{k+q}, q \in \mathbb{Z}$, on the grid.

On the contrary, setting the $\mathbf{q}$ component $\kappa_{3}$ corresponding to the growth direction $\mathbf{a}_{3}$ in the reciprocal basis to zero, (1) examines the 2D structure of all the $M$ bilayers simultaneously. If they are assumed to be identical without lateral ${ }^{2}$ shifts the scattering amplitudes of the bilayer's lattice sites are $2 \mathrm{D}$ real data sets $X_{k_{1} k_{2}}$ with the discrete Fourier transform

$$
S_{r_{1} r_{2}}=M \sum_{k_{1}=0}^{n_{1}-1} \sum_{k_{2}=0}^{n_{2}-1} \exp \left(\frac{2 \pi \mathrm{i} k_{1} r_{1}}{n_{1}}\right) \exp \left(\frac{2 \pi \mathrm{i} k_{2} r_{2}}{n_{2}}\right) X_{k_{1} k_{2}}
$$

for fixed $r_{1}=0,1, \ldots, n_{1}-1$ and $r_{2}=0,1, \ldots, n_{2}-1$ discretizing $\kappa_{1}$ and $\kappa_{2}$. Squaring (10) yields Toeplitz matrices with Kronecker product structure (A.7) for the detected signal

$$
\left|S_{r_{1} r_{2}}\right|^{2}=M^{2} \cdot\left\langle\mathbf{x} \| T_{r_{1}} \otimes T_{r_{2}} \mid \mathbf{x}\right\rangle
$$

with multiple row and column indices $\left(p_{1} p_{2}\right)$ and $\left(q_{1} q_{2}\right)$, respectively, referring to the ordinary rows $p_{1}, p_{2}$ and columns $q_{1}, q_{2}$ of the Toeplitz matrices (9):

$$
\begin{aligned}
\left(T_{r_{1}} \otimes T_{r_{2}}\right)_{\left(p_{1} p_{2}\right),\left(q_{1} q_{2}\right)} & =\left(T_{r_{1}}\right)_{p_{1} q_{1}}\left(T_{r_{2}}\right)_{p_{2} q_{2}}, \\
(\mathbf{x})_{\left(q_{1} q_{2}\right)} & :=X_{q_{1} q_{2}} .
\end{aligned}
$$

The scattering amplitudes in Fourier domain can be assigned with arbitrary phases. As this leaves the given intensity distribution $|S|^{2}$ unchanged an related infinite set of broadened scattering data is formed by convolution. But if the signal $|S|^{2}$ is assumed to be oversampled the original scattering data in this set appear to be sparse and can be recovered by an $\ell_{1}$ minimization run on, for example, (8) or (11) - up to translations, reflections, or global phases.

\section{Kalman Filter-Driven $\ell_{1}$ Minimization}

3.1. Kalman Filter Equations. The equations for the Kalman filter usually apply to vectors over the field of real numbers $\mathbb{R}$. We will examine next that the equations can also be extended to the field $\mathbb{C}$. Anticipating this in the classical state space 
approach [36] the vectorial quantity $\mathbf{x}_{k}$ is supposed to evolve according to the linear evolution

$$
\begin{array}{r}
\mathbf{x}_{k+1}=A_{k} \mathbf{x}_{k}+\mathbf{u}_{k}+G \mathbf{w} \in \mathbb{C}^{n} \text { stochastic } \\
A_{k} \in \mathbb{C}^{n \times n}, \mathbf{u}_{k} \in \mathbb{C}^{n} \text { deterministic } \\
\quad G \in \mathbb{C}^{n \times r}, \mathbf{w} \in \mathbb{C}^{r} \text { stochastic }
\end{array}
$$

with a fixed matrix $G$ and a determined sequence of evolution matrices $A_{k}$. In the model above the quantity $\mathbf{x}_{k}$ is assumed to be only traceable indirectly by linear observations $\mathbf{y}_{k}$ which can be viewed as linear mappings with given sensing matrices $C_{k}$ by

$$
\begin{aligned}
& \mathbf{y}_{k}=C_{k} \mathbf{x}_{k}+\mathbf{v} \in \mathbb{C}^{m} \text { stochastic } \\
& C_{k} \in \mathbb{C}^{m \times n}, \mathbf{v} \in \mathbb{C}^{m} \text { stochastic. }
\end{aligned}
$$

The stochastic behaviour of all the considered quantities is modelled by zero-mean Gaussian distributions with positive definite matrices $R$ and $Q$. These covariance matrices and related mean values can be calculated from normalized Gaussian integrals denoted by expectation or mean values $E\{0\}$ :

$$
\begin{aligned}
E\{\mathbf{w}\} & =\mathbf{0} \in \mathbb{C}^{r}, \\
\operatorname{Cov}\{\mathbf{w}\} & =E\{|\mathbf{w}\rangle\langle\mathbf{w}|\}=Q \in \mathbb{C}^{r \times r}, \\
E\{\mathbf{v}\} & =\mathbf{0} \in \mathbb{C}^{m}, \\
\operatorname{Cov}\{\mathbf{v}\} & =E\{|\mathbf{v}\rangle\langle\mathbf{v}|\}=R \in \mathbb{C}^{m \times m} .
\end{aligned}
$$

The main idea behind Kalman filtering is to invert the observation model (14a) and (14b), where the estimation of the quantities $\mathbf{x}_{k}$ from the measurements $\mathbf{y}_{k}$ shows predictorcorrector structure [36]: the prediction step relates the estimates $\mathbf{x}_{k+1}^{-}$and $\mathbf{x}_{k}^{+}$by extrapolation

$$
\begin{aligned}
\mathbf{x}_{k+1}^{-} & =A_{k} \mathbf{x}_{k}^{+}+\mathbf{u}_{k}, \\
P_{k}^{+} & =\operatorname{Cov}\left\{\mathbf{x}_{k}^{+}\right\}, \\
P_{k+1}^{-} & =A_{k} P_{k}^{+} A_{k}^{H}+G Q G^{H},
\end{aligned}
$$

whereas the correction step updates the estimate $\mathbf{x}_{k+1}^{-}$by relating it to the new measurement $\mathbf{y}_{k+1}$ : as the underlying equations utilize conditional mean values the update can be formulated in terms of covariances

$$
\begin{aligned}
\left(P_{k+1}^{+}\right)^{-1} & =\left(P_{k+1}^{-}\right)^{-1}+C_{k+1}^{H} R^{-1} C_{k+1}, \\
\mathbf{x}_{k+1}^{+} & =P_{k+1}^{+}\left(P_{k+1}^{-}\right)^{-1} \mathbf{x}_{k+1}^{-}+P_{k+1}^{+} C_{k+1}^{H} R^{-1} \mathbf{y}_{k+1} .
\end{aligned}
$$

An alternative form of the covariance cycle in the correction step above reads, in terms of the explicit so-called Kalman gain matrix $K_{k+1}$,

$$
\begin{aligned}
K_{k+1} & =P_{k+1}^{-} C_{k+1}^{H}\left(C_{k+1} P_{k+1}^{-} C_{k+1}^{H}+R\right)^{-1}, \\
P_{k+1}^{+} & =\left(\mathbb{1}-K_{k+1} C_{k+1}\right) P_{k+1}^{-}, \\
\mathbf{x}_{k+1}^{+} & =\mathbf{x}_{k+1}^{-}+K_{k+1}\left(\mathbf{y}_{k+1}-C_{k+1} \mathbf{x}_{k+1}^{-}\right) .
\end{aligned}
$$

3.2. $\ell_{1}$ Minimization. The main problem in CS is to minimize $\|\mathbf{x}\|_{1}$ subject to the constraint $\mathbf{y}=C \mathbf{x}$ for fixed $\mathbf{y}$ and $C$. Because this linear structure matches the evolution equation (13a) of Kalman filtering in Section 3.1 we want to follow [29] using a pseudomeasurement to iteratively minimize the $\ell_{1}$ norm: here, the $k$ th estimate of the state vector $\mathbf{x}$ can be associated with $\mathbf{x}_{k}$ and the norm minimization is driven by the fixed constraint $\mathbf{y}$ augmented by the lowered norm $\gamma\left\|\mathbf{x}_{k-1}\right\|_{1}$ from the previous step by a factor of $0<\gamma \leq 1$ as an additional observation. The main issue of applying the Kalman filter to the $\ell_{1}$ minimization procedure consists in suitably linearizing the nonanalytic $\ell_{1}$ norm: Solving

$$
|z|^{2}=\operatorname{Re}^{2}\left(\frac{z \overline{z_{0}}}{\left|z_{0}\right|}\right)+\operatorname{Im}^{2}\left(\frac{z \overline{z_{0}}}{\left|z_{0}\right|}\right)
$$

for $|z|$ in the vicinity of $z_{0} \in \mathbb{C}$ yields for $\varphi:=\arg \left(z \overline{z_{0}} /\left|z_{0}\right|\right)$ the expression

$$
|z|=\operatorname{Re}\left(\frac{z \overline{z_{0}}}{\left|z_{0}\right|}\right) \sqrt{1+\tan ^{2} \varphi} \geq \operatorname{Re}\left(\frac{z \overline{z_{0}}}{\left|z_{0}\right|}\right) .
$$

Thus the $\ell_{1}$ norm of any vector $\mathbf{z} \in \mathbb{C}^{n}$ can be linearized in the vicinity of $\mathbf{z}_{0} \in \mathbb{C}^{n}$ utilizing its phase information $\mathbf{p} \in \mathbb{C}^{n}$ by

$$
\begin{aligned}
\operatorname{Re}\langle\mathbf{p} \mid \mathbf{z}\rangle & \leq\|\mathbf{z}\|_{1}, \\
\langle\mathbf{p}| & =\langle\mathbf{p}|\left(\mathbf{z}_{0}\right):=\left(\frac{\left(\overline{\mathbf{z}_{0}}\right)_{1}}{\left|\left(\mathbf{z}_{0}\right)_{1}\right|}, \ldots, \frac{\left(\overline{\mathbf{z}_{0}}\right)_{n}}{\left|\left(\mathbf{z}_{0}\right)_{n}\right|}\right) .
\end{aligned}
$$

Due to vectors over the field $\mathbb{C}$ it is necessary to distinguish row vectors $\langle\mathbf{p}|$ from the usual column vectors $|\mathbf{p}\rangle:=$ $\mathbf{p}$ related by Hermitian conjugation. The notation is based on complex scalar products and matrix multiplication (cf. Appendix A).

3.3. Pseudomeasurements. Involving the observation $\mathbf{y}=$ $C \mathbf{x} \in \mathbb{C}^{m}$ as a constraint like, for example, a Fourier transform, the Kalman filter equations (16a), (16b), (17a), and (17b) read (for $A=G=\mathbb{1}, \mathbf{u}_{k}=\mathbf{0}$ ) for the estimates $\mathbf{x}_{k}:=\mathbf{x}_{k}^{+}$ to the vector $\mathbf{x}$ involving a full prediction-correction step

$$
\begin{aligned}
& P_{k+1}^{-1}=\left(P_{k}+Q\right)^{-1}+C_{k+1}^{H} R^{-1} C_{k+1} \\
& \mathbf{x}_{k+1}=P_{k+1} P_{k}^{-1} \mathbf{x}_{k}+P_{k+1} C_{k+1}^{H} R^{-1} \mathbf{y}_{k+1}
\end{aligned}
$$

with the initial values $P_{0} \in \mathbb{C}^{n \times n}, \mathbf{x}_{0} \in \mathbb{C}^{n}$ and the constant parameters $C \in \mathbb{C}^{m \times n}, R \in \mathbb{C}^{(m+1) \times n}$ and $Q \in \mathbb{C}^{n \times n}$ to reconstruct $\mathbf{x}=\lim _{k \rightarrow \infty} \mathbf{x}_{k}$ from a given $\mathbf{y}$ as a limiting value. The minimization of the $\ell_{1}$ norm is incorporated into the (pseudo)measurements by

$$
\begin{gathered}
\mathbf{y}_{k+1}=\left(\frac{\mathbf{y}}{\gamma_{k}\left\|\mathbf{x}_{k}\right\|_{1}}\right) \in \mathbb{C}^{m+1}, \\
C_{k+1}=\left(\frac{C}{\langle\mathbf{p}|\left(\mathbf{x}_{k}\right)}\right) \in \mathbb{C}^{(m+1) \times n},
\end{gathered}
$$


where the factor $0<\gamma_{k} \leq 1$ adaptively lowers the current $\ell_{1}$ norm solving for a corresponding estimate $\mathbf{x}_{k+1}$ in the next iteration step. For a solution to the linear constraint $C \in \mathbb{C}^{m \times n}$ as initial data can serve

$$
\mathbf{x}_{0}= \begin{cases}\left(C^{H} C\right)^{-1} C^{H} \mathbf{y} & \text { for } m>n \\ C^{H}\left(C C^{H}\right)^{-1} \mathbf{y} & \text { for } m \leq n .\end{cases}
$$

3.4. Convergence Considerations. By inserting (22a) into (22b) the enhancement of the estimate $\mathbf{x}_{k+1}$ compared to $\mathbf{x}_{k}$ reads with respect to the measurement $\mathbf{y}_{k+1} \in \mathbb{C}^{m+1}$ of a lowered $\ell_{1}$ norm

$$
\begin{aligned}
\left(C_{k+1} \mathbf{x}_{k+1}-\mathbf{y}_{k+1}\right)= & {\left[R-C_{k+1} P_{k+1} C_{k+1}^{H}\right] R^{-1} } \\
& \cdot\left(C_{k+1} \mathbf{x}_{k}-\mathbf{y}_{k+1}\right) .
\end{aligned}
$$

Using the covariance cycle (22a) again the enhancement matrix composed of positive definite matrices $R, P$, and $Q$ on the RHS can be recast into the more suitable ${ }^{3}$ form

$$
\begin{aligned}
& {\left[R-C_{k+1} P_{k+1} C_{k+1}^{H}\right] R^{-1}} \\
& \quad=R \cdot\left[R+C_{k+1}\left(P_{k}+Q\right) C_{k+1}^{H}\right]^{-1} \leq \mathbb{1}_{m+1}
\end{aligned}
$$

yielding the inequality

$$
\begin{array}{r}
\left|\left\langle\mathbf{e}_{j} \mid C_{k+1} \mathbf{x}_{k+1}-\mathbf{y}_{k+1}\right\rangle\right| \leq\left|\left\langle\mathbf{e}_{j} \mid C_{k+1} \mathbf{x}_{k}-\mathbf{y}_{k+1}\right\rangle\right|, \\
j=1, \ldots, m+1,
\end{array}
$$

by components. The key ingredients are the restricted positive eigenvalues of (26) bounded from above by the spectral norm $\left\|1_{m+1}\right\|_{2}=1$ (cf. (B.1)). What remains to prove for the inequality in (26) is the combination $C_{k}(P+Q) C_{k}^{H}$ to be positive semidefinite.

In the overdetermined case $m \geq n$ this holds true for $C_{k}^{H} C_{k}$ to be positive definite: as the combination $C_{k}(P+$ Q) $C_{k}^{H} \in \mathbb{C}^{(m+1) \times(m+1)}$ has only rank $n$ the amount of $m+1-n$ of its eigenvalues is zero. The positive sign of the remaining eigenvalues can be obtained from a Cholesky factorization of $P+Q=F F^{H}$ with a lower triangular matrix $F \in \mathbb{C}^{n \times n}$ yielding $C_{k} F F^{H} C_{k}^{H}=\left(C_{k} F\right)\left(C_{k} F\right)^{H}$. Due to a singular value decomposition [37] of $C_{k} F$ the remaining eigenvalues belong to $\left(C_{k} F\right)^{H}\left(C_{k} F\right)=F^{H}\left(C_{k}^{H} C_{k}\right) F \in \mathbb{C}^{n \times n}$ which, however, is along with the prerequisite $C_{k}^{H} C_{k}>0$ positive definite because of Theorem 1 .

Theorem 1 (see [37]). If $B \in \mathbb{C}^{n \times n}$ is positive definite and $X \in$ $\mathbb{C}^{n \times k}$ has rank $k$, then the matrix $Y=X^{H} B X \in \mathbb{C}^{k \times k}$ is also positive definite.

By virtue of the same theorem the combination $C_{k}(P+$ Q) $C_{k}^{H} \in \mathbb{C}^{(m+1) \times(m+1)}$ is always positive definite in the underdetermined case $m<n$, which is independent of the sensing matrix $C_{k}$.

So in each iteration step with adaptive $0<\gamma_{k} \leq 1$ the linearized $\ell_{1}$ norm is lowered (or remains at least constant) subject to the approximated constraint $\mathbf{y}=C_{k} \mathbf{x}_{k}$. In the real case (20) even holds with " $=$ " yielding for $j=m+1$ the relation $\left|\left\|\mathbf{x}_{k+1}\right\|_{1}-\gamma_{k}\left\|\mathbf{x}_{k}\right\|_{1}\right| \leq\left(1-\gamma_{k}\right)\left\|\mathbf{x}_{k}\right\|_{1}$ implying $\left\|\mathbf{x}_{k+1}\right\|_{1} \leq$ $\left\|\mathbf{x}_{k}\right\|_{1}$ for all $\gamma_{k}$. To show a general convergence let us start with the exact solution $\mathbf{x}_{\infty}=\mathbf{x}$ and $C_{\infty}$ related to it. Because of $A=G=\mathbb{1}$ with zero shifts $\mathbf{u}_{k}=\mathbf{0}$ in the Kalman filter model (13a), (13b), and (13c) the constant (pseudo)measurement $\mathbf{y}_{\infty}$ already implies with $\gamma_{k} \equiv 1$ a linear convergence of the series $\left\{C_{\infty} \mathbf{x}_{0}, C_{\infty} \mathbf{x}_{1}, C_{\infty} \mathbf{x}_{2}, \ldots\right\}$ by

$$
\begin{aligned}
& \left|\left\langle\mathbf{e}_{j} \mid C_{\infty} \mathbf{x}_{k+1}-\mathbf{y}_{\infty}\right\rangle\right| \leq\left|\left\langle\mathbf{e}_{j} \mid C_{\infty} \mathbf{x}_{k}-\mathbf{y}_{\infty}\right\rangle\right| \leq \cdots \\
& \quad \leq\left|\left\langle\mathbf{e}_{j} \mid C_{\infty} \mathbf{x}_{0}-\mathbf{y}_{\infty}\right\rangle\right|
\end{aligned}
$$

for all $j=1, \ldots, m+1$ starting with an $\mathbf{x}_{0}$ in the vicinity of $\mathbf{x}_{\infty}$. Thus the series $\left\{\mathbf{x}_{0}, \mathbf{x}_{1}, \mathbf{x}_{2}, \ldots\right\}$ is said to be weakly convergent and reconstructs the original signal as long as the requirement for sparsity in the CS approach is met. In the framework of weak convergence the limiting value of $\operatorname{Cov}\left\{\mathbf{x}_{k}\right\}$ reads for large $k \gg 1$

$$
\begin{aligned}
& P_{k} \\
& \geq \begin{cases}P_{0}-P_{0} C_{\infty}^{H}\left(C_{\infty} P_{0} C_{\infty}^{H}\right)^{-1} C_{\infty} P_{0}+\mathcal{O}\left(\frac{1}{k}\right), & m<n \\
\frac{1}{k}\left(C_{\infty}^{H} R^{-1} C_{\infty}\right)^{-1}+\mathcal{O}\left(\frac{1}{k^{2}}\right), & m \geq n,\end{cases}
\end{aligned}
$$

where "=" represents the special case $Q \equiv 0$. The limit $Q \rightarrow$ $\infty$ is only possible for $m \geq n$ yielding a constant covariance $P_{k}=\left(C_{\infty}^{H} R^{-1} C_{\infty}\right)^{-1}$ in each iteration.

For an example of linear compressive sensing in the framework of Kalman filtering see [30, 31] using random samples of Fourier coefficients. Applying the method to a quadratic nonlinear observation model is shown for coherent $\mathrm{X}$-ray scattering in Section 5.

\section{Linearized Observation Model}

Like the $\ell_{1}$ norm in Section 3.2 we linearize the squared observations (8) to apply the Kalman filtering scheme. Differentiating with respect to $x_{k}$ and $\overline{x_{k}}$, respectively, yields

$$
\begin{aligned}
& \frac{\partial}{\partial x_{k}}\left|S_{r}\right|^{2}=\left(\left\langle\mathbf{x} \| T_{r}\right)_{k},\right. \\
& \frac{\partial}{\partial \overline{x_{k}}}\left|S_{r}\right|^{2}=\left(T_{r}|\mathbf{x}\rangle\right)_{k}
\end{aligned}
$$

and Taylor expansion around $\mathbf{z}_{0} \in \mathbb{C}^{n}$ reads up to 1st order

$$
\begin{aligned}
\left|S_{r}\right|^{2}=2 \operatorname{Re}\left\langle\mathbf{z}_{0} \| T_{r} \mid \mathbf{x}\right\rangle-\left\langle\mathbf{z}_{0} \| T_{r} \mid \mathbf{z}_{0}\right\rangle & +\cdots, \\
r & =0,1, \ldots, n-1 .
\end{aligned}
$$


With the biases $s_{r}=\left\langle\mathbf{z}_{0} \| T_{r} \mid \mathbf{z}_{0}\right\rangle, r=0,1, \ldots, n-1$, from the linearization the observation model is

$$
\begin{aligned}
&\left(\begin{array}{c}
\left|S_{0}\right|^{2} \\
\vdots \\
\left|S_{n-1}\right|^{2} \\
\|\mathbf{x}\|_{1}
\end{array}\right)=\left(\begin{array}{c}
2\left\langle\mathbf{z}_{0} \| T_{0}\right. \\
\vdots \\
2\left\langle\mathbf{z}_{0} \| T_{n-1}\right. \\
\langle\mathbf{p}|\left(\mathbf{z}_{0}\right)
\end{array}\right) \cdot\left(\begin{array}{c}
x_{0} \\
\vdots \\
x_{n-2} \\
x_{n-1}
\end{array}\right) \\
&-\left(\begin{array}{c}
s_{0} \\
\vdots \\
s_{n-1} \\
0
\end{array}\right) \\
& \mathbf{y}_{k}=C_{k} \cdot \mathbf{x}-\mathbf{s}_{k},
\end{aligned}
$$

where the real parts over all $C \mathbf{x}$ combinations have to be taken. The model in detail reads $\mathbf{y}_{k}=\operatorname{Re}\left(C_{k} \mathbf{x}\right)-\mathbf{s}_{k} \in \mathbb{R}^{n+1}$ and because of the $C \mathbf{x}$ combinations we choose the alternative representation (18a), (18b), and (18c) of the Kalman filter equations to invert (32). Then the iterated estimates $\mathbf{x}_{k}$ to the state vector $\mathbf{x}$ read

$$
\begin{aligned}
K_{k+1} & =\left(P_{k}+Q\right) C_{k+1}^{H}\left(C_{k+1}\left(P_{k}+Q\right) C_{k+1}^{H}+R\right)^{-1}, \\
P_{k+1} & =\left(\mathbb{1}-K_{k+1} C_{k+1}\right)\left(P_{k}+Q\right), \\
\mathbf{x}_{k+1} & =\mathbf{x}_{k}+K_{k+1} \cdot \operatorname{Re}\left[\mathbf{y}_{k+1}-C_{k+1} \mathbf{x}_{k}+\mathbf{s}_{k+1}\right]
\end{aligned}
$$

with the complex sensing matrix $C_{k+1} \in \mathbb{C}^{(n+1) \times n}$, biases $\mathbf{s}_{k+1} \in \mathbb{C}^{n+1}$, and the given real (pseudo)measurements $\mathbf{y}_{k+1} \in$ $\mathbb{R}^{n+1}$ according to

$$
\begin{aligned}
C_{k+1}= & \left(\begin{array}{c}
2\left\langle\mathbf{x}_{k} \| T_{0}\right. \\
\vdots \\
2\left\langle\mathbf{x}_{k} \| T_{n-1}\right. \\
\langle\mathbf{p}|\left(\mathbf{x}_{k}\right)
\end{array}\right), \\
\mathbf{s}_{k+1}= & \left(\begin{array}{c}
\left\langle\mathbf{x}_{k} \| T_{0} \mid \mathbf{x}_{k}\right\rangle \\
\vdots \\
\left\langle\mathbf{x}_{k} \| T_{n-1} \mid \mathbf{x}_{k}\right\rangle \\
0 \\
\left|S_{0}\right|^{2} \\
\vdots \\
\left|S_{n-1}\right|^{2} \\
\gamma_{k}\left\|\mathbf{x}_{k}\right\|_{1}
\end{array}\right),
\end{aligned}
$$

A properly chosen factor $0<\gamma_{k} \leq 1$ adaptively lowers the $\ell_{1}$ norm in each iteration step $k$ to reconstruct the signal $\mathbf{x}=\lim _{k \rightarrow \infty} \mathbf{x}_{k}$ as a limiting value from its given squared measurements $\langle\mathbf{y}|=\left(\left|S_{0}\right|^{2}, \ldots,\left|S_{n-1}\right|^{2}\right)$. Note that $\left\{T_{0}\left|\mathbf{x}_{k}\right\rangle, T_{1}\left|\mathbf{x}_{k}\right\rangle, \ldots, T_{n-1}\left|\mathbf{x}_{k}\right\rangle\right\} \subset \mathbb{C}^{n}$ form an orthogonal basis. Thus

$$
C_{k+1}^{H} C_{k+1}=4 \sum_{r=0}^{n-1} T_{r}\left|\mathbf{x}_{k}\right\rangle\left\langle\mathbf{x}_{k} \| T_{r}+\mid \mathbf{p}\right\rangle\langle\mathbf{p}|\left(\mathbf{x}_{k}\right)
$$

is Hermitian and positive definite due to (B.1) for all $k$ with $\left|\mathbf{x}_{k}\right\rangle \neq|\mathbf{0}\rangle$ which is sufficient to prove weak convergence of the $\ell_{1}$ minimization under the constraint of squared observations (cf. (26)-(28)).

4.1. Example 1: Stacking Sequence. To demonstrate the relative phase recovery from the observed intensity (8) not only restricting to the Zinc-Blende and Wurtzite phases (6) assume a linear chain with 117 lattice sites and 6 equidistant sparse amplitudes $\left|x_{j}\right|=1$ with phases from the set $\{-\pi / 3,-\pi / 6,0, \pi / 6, \pi / 3, \pi / 2\}$ forming $\mathbf{x}_{\text {sparse }} \in \mathbb{C}^{n}$.

As in the original objective the number of bilayers is roughly known we use as initial values $\mathbf{x}_{0} \in \mathbb{C}^{n}$ a broadened modulus and phase distribution related to the setting $\mathbf{x}_{\text {sparse }}$ (cf. Figure 3): with the support $\mathbb{S}:=\operatorname{supp} \mathbf{x}_{\text {sparse }}$ a leakage

$$
\left(\mathbf{f}_{0}^{ \pm}\right)_{k}=\sum_{\alpha \in \mathbb{S}} \frac{\sin ((k \pm s-\alpha) \pi)}{(k \pm s-\alpha) \pi} \in \mathbb{R}, \quad k=0, \ldots, n-1
$$

for the moduli and phases of $\mathbf{x}_{\text {sparse }}$ is with shifts $0 \leq s<1$, a suitable way to also consider the limit $s \rightarrow 0$ of exactly known positions. Note that for the phases we only used one fixed value for each occupied lattice site broadened by the leakage. So the main effort is the recovery of the relative phases from the measurements.

For an exponential decaying lowering factor $\gamma_{k}=1-$ $0.1 \cdot \exp (-0.0019 k)$ within 1200 iterations the reconstruction results for noiseless synthetic measurements are shown in Figures 4 and 5. Experientially, good algorithm's covariances were found to be

$$
\begin{aligned}
& P_{0}=0.3 \cdot \mathbb{1}_{117}, \\
& Q=10^{-8} \cdot \mathbb{1}_{117}, \\
& R=\operatorname{diag}\left(10^{-4}, \ldots, 10^{-4}, 10^{-6}\right) \in \mathbb{R}^{118 \times 118} .
\end{aligned}
$$

To drive the $\ell_{1}$ minimization the corresponding entry (here $10^{-6}$ ) in the $R$ matrix has to be much lower in magnitude than the ones (here $10^{-4}$ ) related to the observations of the constraint. When reconstructed amplitudes are said to vanish (e.g., in Figure 4) this means only up to numerical precision where the zero value is dominated by the entries of Q. Empirically, the exponential decay in the lowering factor $\gamma_{k}$ is chosen such that its values approach about $0.99, \ldots, 0.999$ after the maximum number of iterations. A resulting typical smooth convergence of the $\ell_{1}$ norm due to the exponential decay is shown in Figure 6: the constant tail can be used to determine a maximal number of iterations as a stopping criterion. Note that the reconstructed results beyond this guessed number are insensitive to further iterations meaning for (34a), (34b), and (34c) to perform fixed point iterations within accuracies related to the covariances $Q$ and $P_{k}$. 


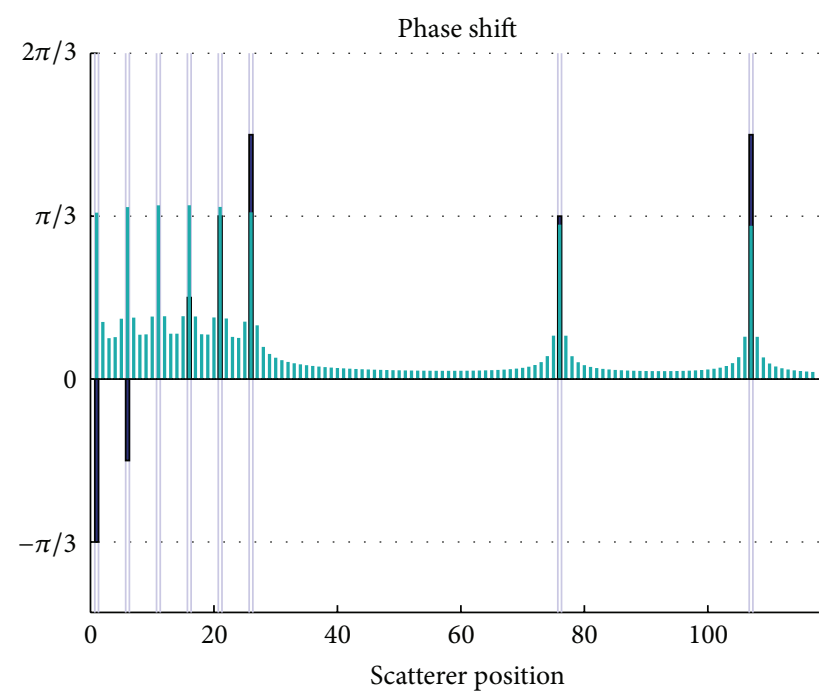

$\square$ Scatterer positions

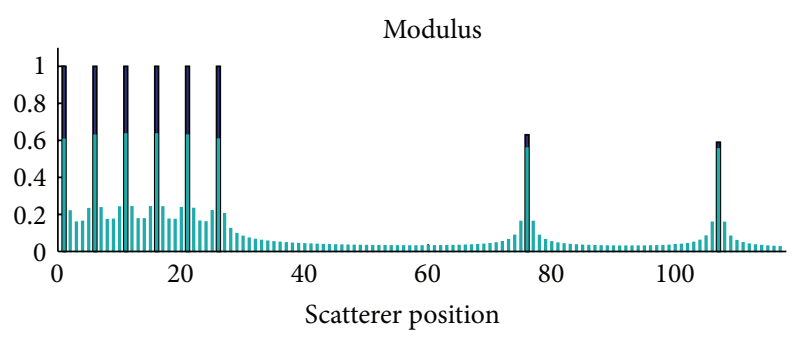

$$
\begin{aligned}
& \text { Actual scatterers } \\
& \text { Initial values }
\end{aligned}
$$

FIGURE 3: A number of 6 equidistant scatterers on a periodic chain of length 117. To allow for a signal from the substrate the wires are growing on we added some parasitic scatterers to the chain. As initial data we use the moduli and phases from $\mathbf{x}_{\text {sparse }}$ symmetrically broadened by a leakage $(1 / 2)\left(\mathbf{f}_{0}^{+}+\mathbf{f}_{0}^{-}\right)$(cf. (37)) with shift $s=0.3$ and amplitudes 0.6 and 1.1 , respectively.

In the nanowire the equidistant bilayers are at fixed positions in growth direction $\mathbf{a}_{3}$. Thus the reconstruction of their complex scattering amplitudes does not suffer from "off-grid" problems in general (see, e.g., [38-40]) if integer multiples of the lattice constants are used for the DFT.

4.2. Example 2: Pattern Reconstruction. The 2D reconstruction from (11) can be calculated with the same algorithm already used for Example 1 above, as the $2 \mathrm{D}$ data set of size $12 \times 25$ can be mapped to a $1 \mathrm{D}$ vector by means of (12) (cf. Figure 7). Like the example from Figure 3, we use as initial values with the same reasoning and broadening parameters the given distribution. Within 2000 iterations and an exponentially decaying lowering factor $\gamma_{k}=$ $1-0.17 \cdot \exp (-0.0028 k)$ the reconstruction for noiseless measurements is shown in Figure 8 with empirically good algorithm's covariances:

$$
\begin{aligned}
& P_{0}=0.3 \cdot \mathbb{1}_{300}, \\
& Q=10^{-7} \cdot \mathbb{1}_{300}, \\
& R=\operatorname{diag}\left(10^{-4}, \ldots, 10^{-4}, 10^{-6}\right) \in \mathbb{R}^{301 \times 301} .
\end{aligned}
$$
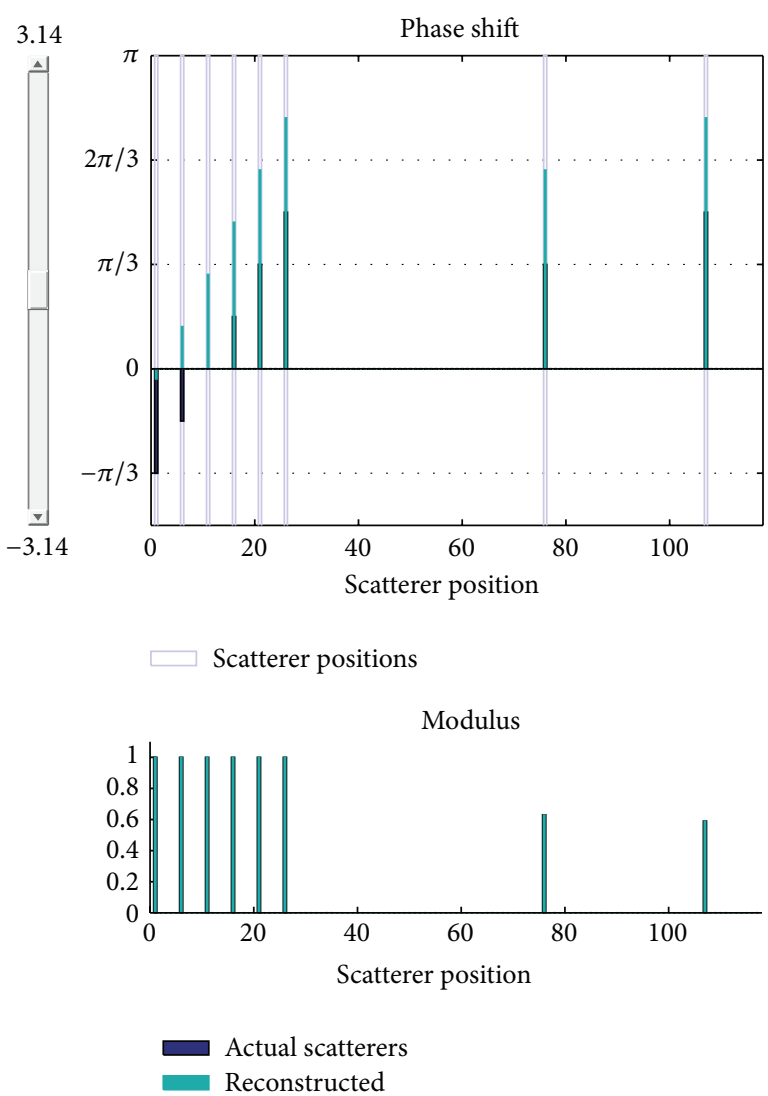

FIGURE 4: Reconstructed moduli of all scatterers. In the phase domain the values corresponding to vanishing moduli are suppressed. Due to the symmetry of the sensing problem the phases are only reconstructed up to a global phase.

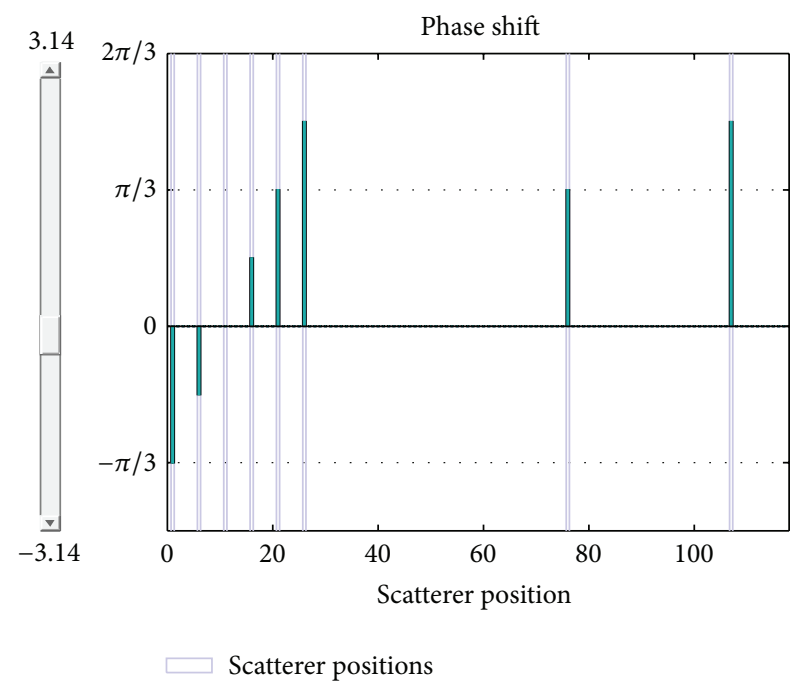

FIGURE 5: Adding a global phase (slider on the left) verifies that all relative phases are reconstructed correctly. The open rectangles mark the position of occupied lattice sites on the chain. In the original objective this would refer to bilayers and substrate in the nanowire.

Note that there is a combined translational and reflectional symmetry mapping thevectorized real scattering amplitude 


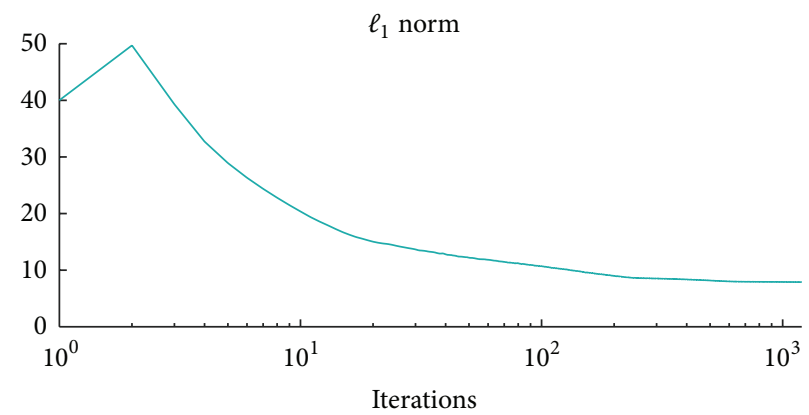

Figure 6: Note that the $\ell_{1}$ convergence (27) in the $k$ th iteration step relates the linearized norm $\left\langle\mathbf{p} \mid \mathbf{x}_{k}\right\rangle \leq\left\|\mathbf{x}_{k}\right\|_{1}$ to $\left\|\mathbf{x}_{k-1}\right\|_{1}$. Because of this inequality (cf. (21)) there is no guarantee of a monotonically lowered $\ell_{1}$ norm, which can be seen in the beginning (iteration step 2).

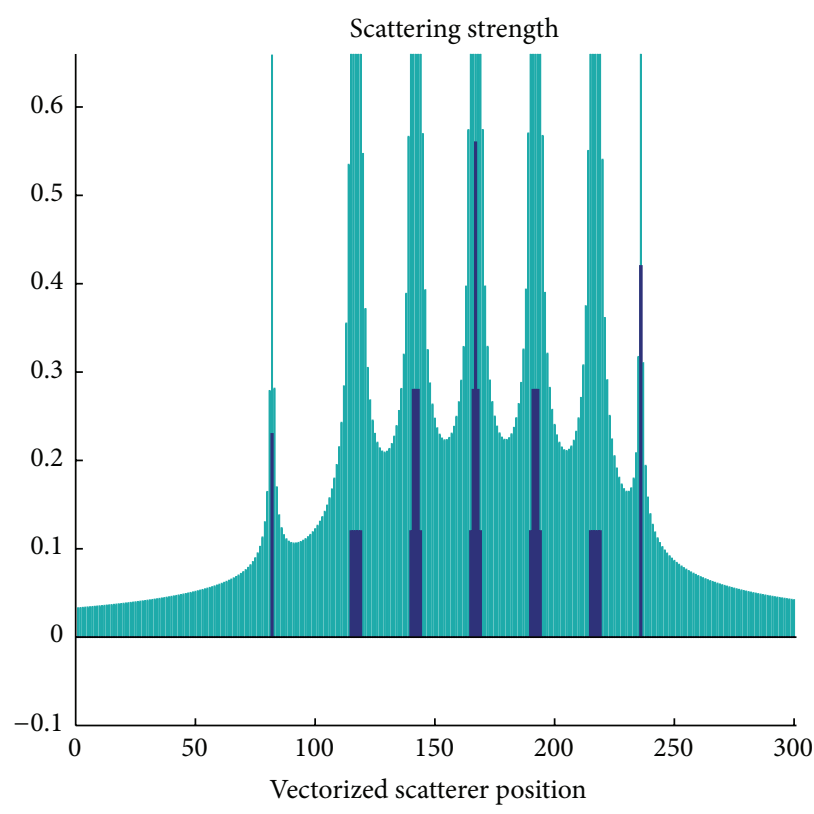

Actual scatterers

Initial values

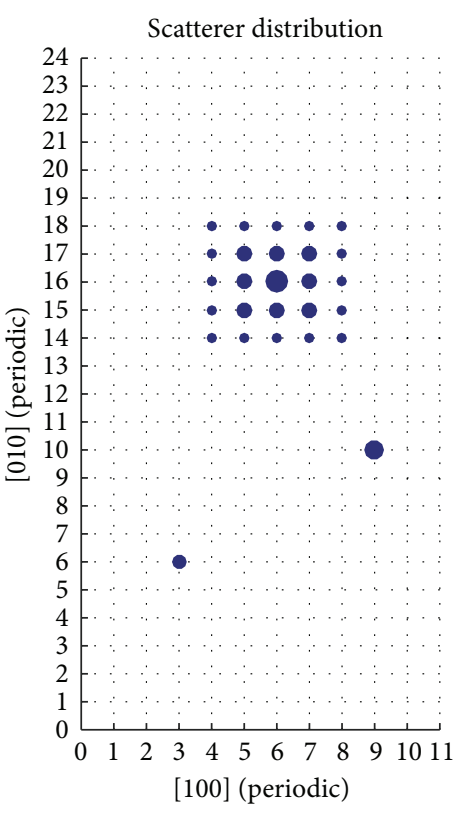

(b)

Figure 7: The actual scatterers forming the $5 \times 5$ structure are made from real amplitudes $0.12,0.28$, and 0.56 whereas the two parasitic scatterers are assigned with 0.23 and 0.42 (a). The scattering strength in the plane is represented by the area of the filled circles (b).

distribution onto itself (cf. Figure 7). As this is also a symmetry of the squared observations (8) in the linear configuration, there are two competing solutions to the sensing problem. Interestingly, breaking this symmetry improved the $\ell_{1}$ convergence in Figure 8: without the parasitic scatterers the symmetric positions of consecutive sites ${ }^{4}$ are correctly (with respect to the support) found after 5000 iterations (cf. Figure 9) and the remaining discrepancies in the amplitudes do not even vanish completely after inefficient 50000 iterations. On the contrary the random sample of 25 scatterers from Figure 10 is already reconstructed with less than 1000 iterations suggesting a strong dependence of the algorithm's convergence from the vector under consideration.

\section{Conclusion and Outlook}

We aimed to implement nonlinear compressive sensing for complex vectors $\mathbf{x}$ by inverting the underlying observation model with Kalman filtering. For this reason we proved a weak convergence of the filter equations for complex sensing matrices $C$ and Hermitian covariances $R, P, Q$. For the example of quadratic nonlinearities we applied our formulas to retrieve relative phase information in the objective of simulated noiseless coherent X-ray diffraction. Due to the nonlinearity the noise in the intensity is not Gaussian and needs further considerations to also account for, for example, lattice distortions or the finite coherence of the primary beam. 


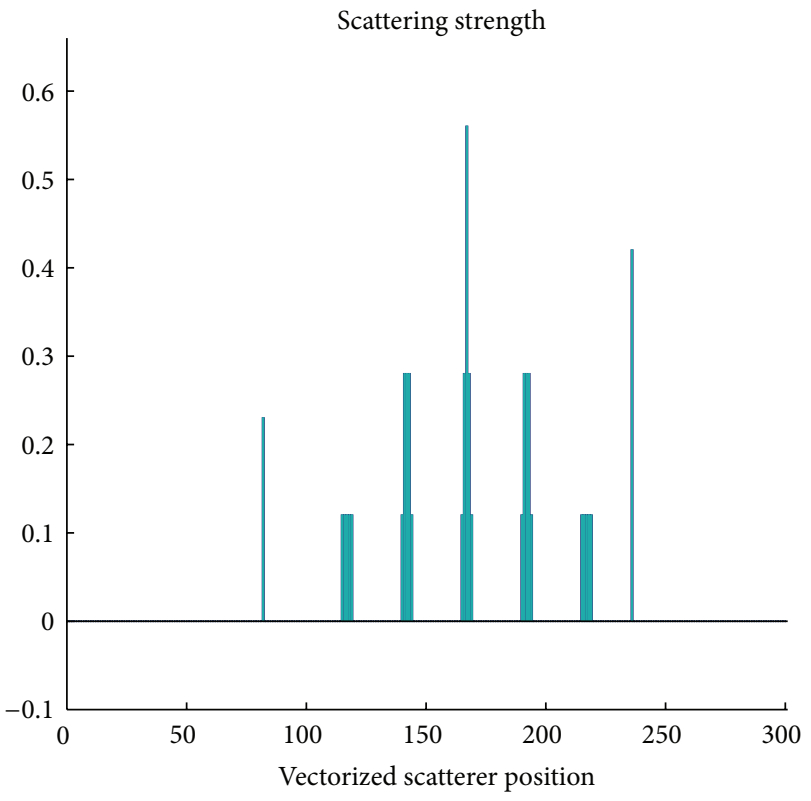

Actual scatterer

Reconstructed

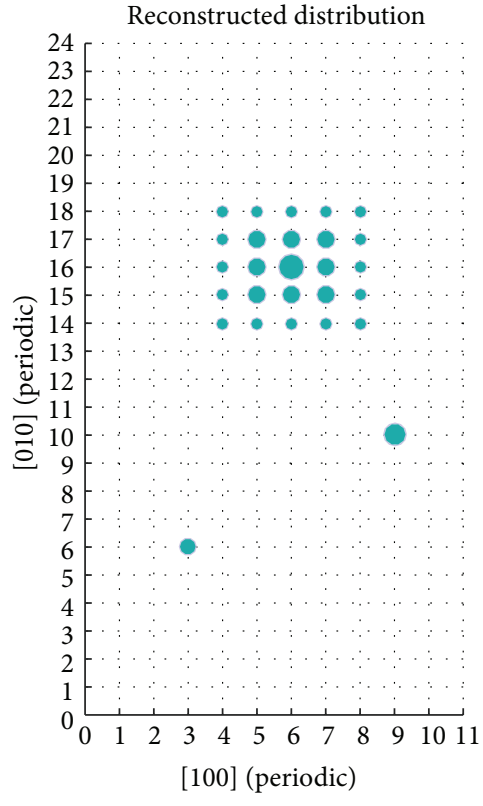

(b)

FIGURE 8: The real amplitudes are nicely reconstructed (a) after 2000 iterations which can also be seen in the 2D setting (b) by comparing with Figure 7.

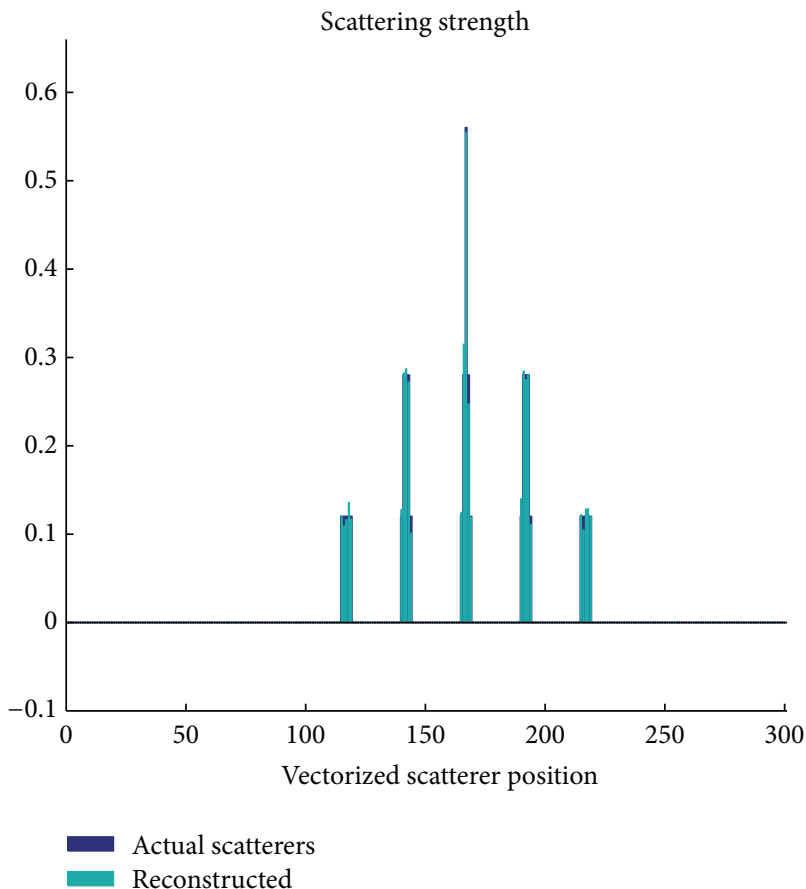

(a)

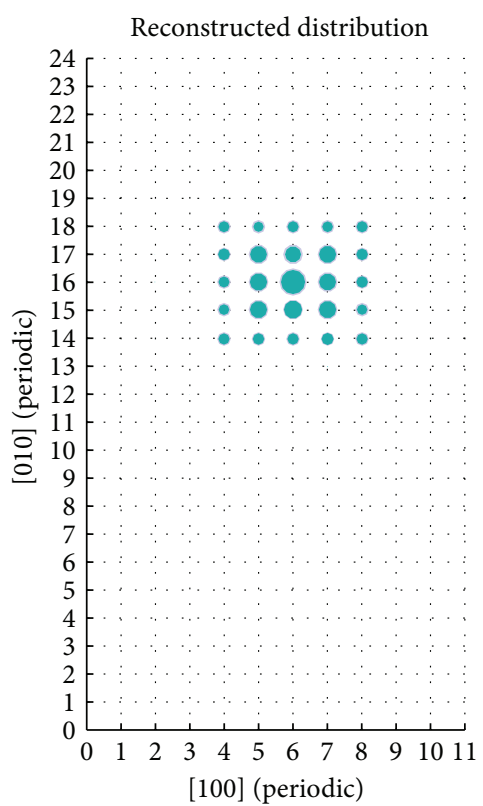

(b)

FIGURE 9: Without parasitic scatterers the positions are correctly found (b) after 5000 iterations and $\gamma_{k}=1-0.17 \cdot \exp (-0.0012 k)$. The remaining discrepancies in the amplitudes seem to be complementarily symmetric with respect to the central peak (a). 

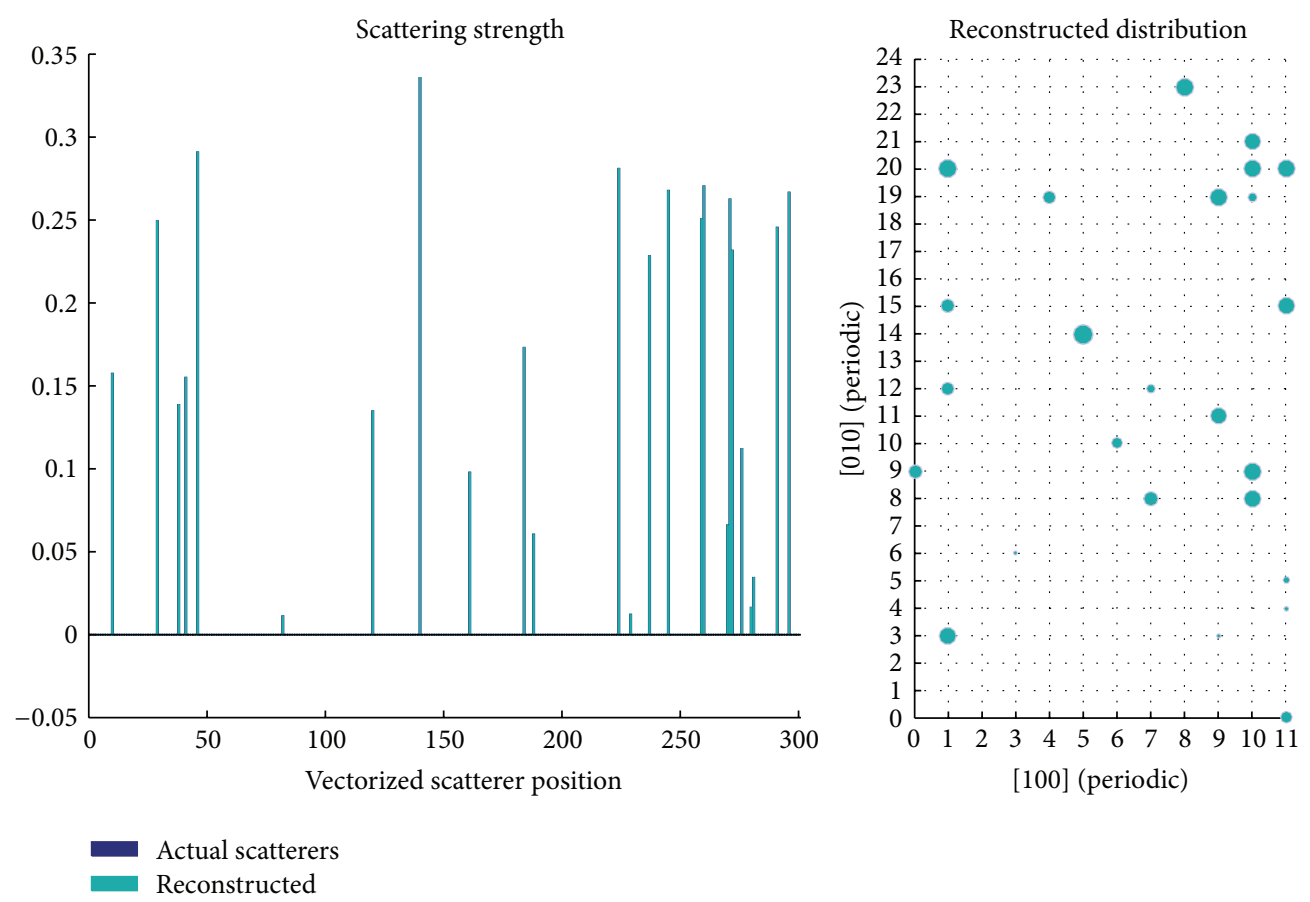

Figure 10: A random distribution of 25 scatterers with real random amplitudes normalized in the $\ell_{2}$ norm. The reconstruction is without artefacts and discrepancies occurred after 1000 iterations with $\gamma_{k}=1-0.17 \cdot \exp (-0.0058 k)$.

As an outlook we presented a $2 \mathrm{D}$ pattern reconstruction which, hopefully, can help to investigate if the cross sections of the wires were grown regularly.

Because of the Jacobians building up the sensing matrix $C$ the convergence of the underlying $\ell_{1}$ norm minimization does depend on the reconstructed vector $\mathbf{x}$ and may go beyond the resolution issues [40] for even constant sensing matrices. For this reason a thorough analysis on the relation between maximal sparsity (for the examples here about $10 \%$ of the available lattice sites) and the Toeplitz matrix (9) representing the sensing process for a successful CS is needed. As mentioned in the introduction algorithms in general minimizing the $\ell_{1}$ norm could be of interest retrieving information out from intensity spectra: to compare our results we applied the nonlinear version [41] of the primal dual algorithm [42] to the $1 \mathrm{D}$ sensing problem above yielding similar results with respect to iteration numbers, reconstructed amplitudes, and phases. On the one hand primal dual reconstructed the zeros outside the support (once a solution was isolated from the algorithm) numerically exact compared to accuracies of orders $10^{-5}$ reached by the Kalman filter. On the other hand our approach focused in the first quarter of the iterations on guessing the support by strongly increasing and decreasing the corresponding amplitudes, whereas primal dual homogeneously acted on all the amplitudes during all iterations. For a detailed comparison and maybe a combination, the whole parameter range of the approaches have to be investigated. To apply the Kalman filter-based algorithm above to recorded data [17] we need to deal with several $10^{4} \mathbf{x}$-vector's entries describing a sparsely (by a factor of roughly $1: 10^{1}$ ) occupied linear grid covering all the approximately $10^{3}$ bilayers in a nanowire of $500 \mathrm{~nm}$ in height. For this reason matrix inversions, cf. (34a), (34b), and (34c), should be reformulated by, for example, sequential processing techniques to make the algorithm more scalable. Furthermore it would also be interesting if other adaptive lowering factors $\gamma_{k}$ compared to the exponential ones yield faster convergences.

As the reconstruction from quadratic constraints seems to depend only little on the explicit algorithm used for the $\ell_{1}$ minimization, it would be interesting to figure out to which extent the linear matrix completion approach [22-24] with respect to the nuclear norm can still be applied in the face of seemingly too few numbers of independent observations.

\section{Appendix}

\section{A. Vector Notations for Complex Numbers}

Vectors over the field $\mathbb{C}$ consist of complex numbers $z=x+$ iy with $x, y \in \mathbb{R}$ and $\mathrm{i}^{2}=-1$. With a vector $|\mathbf{v}\rangle \in \mathbb{C}^{n}$ we usually associate $n$ complex numbers $v_{1}, v_{2}, \ldots, v_{n}$ arranged as a column. With the complex conjugate $\bar{z}:=x-\mathrm{i} y$ row vectors $\langle\mathbf{v}| \in \mathbb{C}^{n}$ are considered to be dual to $|\mathbf{v}\rangle$ by

$$
\begin{aligned}
|\mathbf{v}\rangle & =\left(\begin{array}{c}
v_{1} \\
\vdots \\
v_{n}
\end{array}\right) \in \mathbb{C}^{n}, \\
\langle\mathbf{v}| & :=\left(\overline{v_{1}}, \ldots, \overline{v_{n}}\right) \in \mathbb{C}^{n}, \\
|\mathbf{v}\rangle & =\sum_{k=1}^{n} v_{k}\left|\mathbf{e}_{k}\right\rangle, \\
(|\mathbf{v}\rangle)_{j} & =v_{j} \in \mathbb{C},
\end{aligned}
$$


where $\left(\left|\mathbf{e}_{j}\right\rangle\right)_{k}=\delta_{j k}$ denotes with $j, k=1, \ldots, n$ the usual standard basis. With the complex scalar product

$$
\langle\mathbf{v} \mid \mathbf{w}\rangle=\sum_{k=1}^{n} \overline{v_{k}} w_{k}, \quad|\mathbf{v}\rangle,|\mathbf{w}\rangle \in \mathbb{C}^{n}
$$

each vector $|\mathbf{v}\rangle \in \mathbb{C}^{n}$ can be represented in a unitary basis $\left\{\left|\mathbf{q}_{1}\right\rangle, \ldots,\left|\mathbf{q}_{n}\right\rangle\right\} \subset \mathbb{C}^{n}$ yielding the expansion

$$
\begin{aligned}
|\mathbf{v}\rangle & =\sum_{k=1}^{n}\left|\mathbf{q}_{k}\right\rangle\left\langle\mathbf{q}_{k} \mid \mathbf{v}\right\rangle, \\
\left\langle\mathbf{q}_{j} \mid \mathbf{q}_{k}\right\rangle & =\delta_{j k} \quad \text { with } j, k=1, \ldots, n .
\end{aligned}
$$

With $A=\left(a_{i j}\right)_{i j} \in \mathbb{C}^{m \times n}$ we denote matrices with $m$ rows and $n$ columns consisting of the complex entries $a_{i j}=$ $(A)_{i j}$. Let $\left(A^{H}\right)_{i j}:=\overline{(A)_{j i}}=\overline{a_{j i}}$ represent the Hermitian conjugate; then we get from its column decomposition $A^{H}=$ $\left(\left|a_{1}\right\rangle, \ldots,\left|a_{n}\right\rangle\right) \in \mathbb{C}^{m \times n}$,

$$
A=\left(\begin{array}{c}
\left\langle a_{1}\right| \\
\vdots \\
\left\langle a_{n}\right|
\end{array}\right), \quad\left\langle a_{j}|=| a_{j}\right\rangle^{H} \in \mathbb{C}^{m}
$$

Using such a row decomposition the matrix multiplication of commensurable $A \in \mathbb{C}^{m \times n}$ and $B \in \mathbb{C}^{n \times p}$ can be viewed as $m p$ single-scalar products yielding with $B=\left(\left|\mathbf{b}_{1}\right\rangle, \ldots,\left|\mathbf{b}_{p}\right\rangle\right)$

$$
\begin{aligned}
A B & =C=\left(c_{i k}\right)_{i k} \in \mathbb{C}^{m \times p}, \\
c_{i k} & =\sum_{\ell=1}^{n} a_{i \ell} b_{\ell k}=\left\langle\mathbf{a}_{i} \mid \mathbf{b}_{k}\right\rangle
\end{aligned}
$$

including products $A|\mathbf{x}\rangle \in \mathbb{C}^{m}$ for $B=|\mathbf{x}\rangle \in \mathbb{C}^{n}$. Thus with the identity $\mathbb{1}_{n}=\left(\delta_{i j}\right)_{i j} \in \mathbb{C}^{n \times n}$ unitary matrices $Q=$ $\left(\left|\mathbf{q}_{1}\right\rangle, \ldots,\left|\mathbf{q}_{n}\right\rangle\right) \in \mathbb{C}^{n \times n}$ consist of orthonormal (row and) columns defined by the property

$$
\begin{gathered}
Q Q^{H}=Q^{H} Q=\mathbb{1}_{n}, \\
\left(Q^{H} Q\right)_{j k}=\left\langle\mathbf{q}_{j} \mid \mathbf{q}_{k}\right\rangle=\delta_{j k}, \\
Q^{H}=Q^{-1} .
\end{gathered}
$$

The possibility of multiplying matrices $A=\left(a_{i j}\right)_{i j} \in \mathbb{C}^{m \times n}$ and $B=\left(b_{r s}\right)_{r s} \in \mathbb{C}^{p \times q}$ of arbitrary dimensions is covered by the Kronecker product

$A \otimes B$

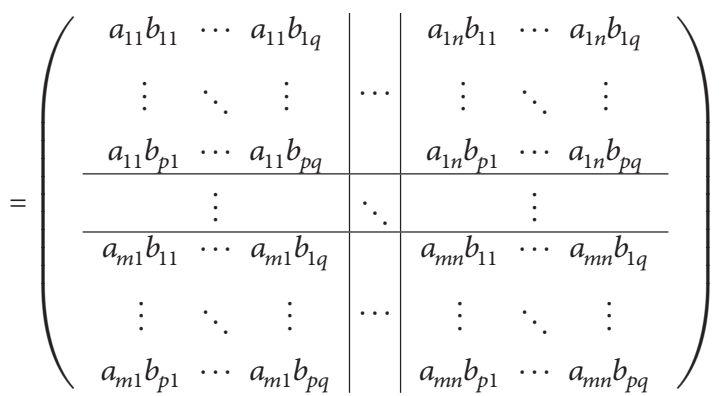

$$
\in \mathbb{C}^{(m p) \times(n q)}
$$

reading in components $(A \otimes B)_{(i r)(j s)}=a_{i j} b_{r s}$ with multiple row and column indices (ir) and (js), respectively. For $|\mathbf{x}\rangle \in$ $\mathbb{C}^{n},|\mathbf{y}\rangle \in \mathbb{C}^{q}$, and $\lambda \in \mathbb{C}$ the Kronecker product meets

$$
\begin{aligned}
(\lambda A|\mathbf{x}\rangle) \otimes(B|\mathbf{y}\rangle) & =(A|\mathbf{x}\rangle) \otimes(\lambda B|\mathbf{y}\rangle) \\
& =\lambda(A \otimes B)(|\mathbf{x}\rangle \otimes|\mathbf{y}\rangle) .
\end{aligned}
$$

Introducing the length of row or column vectors $\mathbf{x} \in \mathbb{C}^{n}$ can be accomplished with the $\ell_{p}$ norms $\|\mathbf{x}\|_{p}$ defined for all real $p>0$ by

$$
\begin{aligned}
& \|\mathbf{x}\|_{p}^{p}:=\sum_{j=1}^{n}\left|x_{j}\right|^{p}, \\
& \|\mathbf{x}\|_{0}:=\operatorname{card}\left\{j \mid x_{j} \neq 0, j=1, \ldots, n\right\} .
\end{aligned}
$$

Particularly $\ell_{0}$ is no norm but can be viewed as the number of nonzero entries. The corresponding matrix norms of $A \in$ $\mathbb{C}^{m \times n}$ can be related to the vector norms according to

$$
\|A\|_{p}:=\sup _{\mathbf{x} \in \mathbb{C}^{n}} \frac{\|A \mathbf{x}\|_{p}}{\|\mathbf{x}\|_{p}}=\sup _{\|\mathbf{x}\|_{p}=1}\|A \mathbf{x}\|_{p} .
$$

\section{B. Hermitian Matrices}

Hermitian matrices $X, Y \in \mathbb{C}^{n \times n}$ can be related by $X>Y(X \geq$ $Y)$ if $X-Y$ is positive (semi)definite which can be written as $X-Y>0(X-Y \geq 0)$. In addition, there is Theorem B.1.

Theorem B.1 (see [43]). If $X, Y \in \mathbb{C}^{n \times n}$ are Hermitian with $X \geq Y>0$, then $0<X^{-1} \leq Y^{-1}$.

Proof. $Y=Y^{1 / 2} Y^{1 / 2}$, cf. $\$ 9.2 .4$ in [37] $\Rightarrow \mathbb{1}_{n} \leq Y^{-1 / 2} X Y^{-1 / 2}$, has eigenvalues larger than $1 \Rightarrow Y^{1 / 2} X^{-1} Y^{1 / 2}$ that has positive eigenvalues lower than $1 \Rightarrow 0<Y^{1 / 2} X^{-1} Y^{1 / 2} \leq \mathbb{1}_{n}$.

Thus all Hermitian matrices $R, X \in \mathbb{C}^{n \times n}$ with $R$ positive definite and $X$ positive semidefinite satisfy the inequalities

$$
\begin{aligned}
R+X & \geq R>0 \Longleftrightarrow \\
0 & <(R+X)^{-1} \leq R^{-1} .
\end{aligned}
$$




\section{Competing Interests}

The authors declare that they have no competing interests.

\section{Acknowledgments}

This work has been supported in part by the Deutsche Forschungsgemeinschaft under Grants Lo 455/20-1 and Pi 214/38-2.

\section{Endnotes}

1. In solid state physics the reciprocal vectors $\mathbf{q}$ according to (3) and (4) for $\kappa_{j}$ are said to be from the 1 st Brillouin zone which is a fragmentation of the elementary cell spanned by $\left\{\mathbf{b}_{1}, \ldots, \mathbf{b}_{d}\right\}$.

2. However, in the hexagonal lattice there are three possible lateral shifts yielding additional phase factors in (10) for each layer. So carrying out the complete Fourier transform of all bilayers yields a random sequence $c_{1}+$ $c_{2}+\cdots+c_{M}$ with

$$
\begin{aligned}
c_{j} & \in\left\{1, \exp \left(\frac{2 \pi \mathrm{i}}{3}\left(\frac{2 r_{1}}{n_{1}}+\frac{r_{2}}{n_{2}}\right)\right),\right. \\
& \left.\exp \left(\frac{2 \pi \mathrm{i}}{3}\left(\frac{r_{1}}{n_{1}}+\frac{2 r_{2}}{n_{2}}\right)\right)\right\}, \quad j=1, \ldots, M
\end{aligned}
$$

rather than the constant $M$ in front of (10) and (11). In the general case the $\mathbf{q}$ vectors corresponding to the bilayers can be expressed by $\mathbf{q}=\kappa_{1} \mathbf{b}_{1}+\kappa_{2} \mathbf{b}_{2}$. Then the phase factors $(*)$ read

$$
\begin{aligned}
c_{j} & \in\left\{1, \exp \left(\frac{2 \pi \mathrm{i}}{3}\left(2 \kappa_{1}+\kappa_{2}\right)\right),\right. \\
& \left.\exp \left(\frac{2 \pi \mathrm{i}}{3}\left(\kappa_{1}+2 \kappa_{2}\right)\right)\right\}, \quad j=1, \ldots, M
\end{aligned}
$$

and their $\mathbf{q}$ dependence can be suppressed by choosing wave vectors related to the Bragg condition by $\kappa_{1}-$ $\kappa_{2}=3 N$ with $N, \kappa_{1}, \kappa_{2} \in \mathbb{Z}$. One could be misled that this selects a certain amount of data points on the grid spanned by $\left\{\mathbf{b}_{1}, \mathbf{b}_{2}\right\}$ allowing for CS techniques. Switching to the discrete version this reduces to one corner of the 1st Brillouin zone, that is, to the case of one single data point $r_{1}=r_{2}=0$ which is in fact too little for CS.

3. A completely factorized version of (26) reads for $m<n$

$$
\begin{aligned}
R \cdot & {\left[R+C_{k+1}\left(P_{k}+Q\right) C_{k+1}^{H}\right]^{-1} } \\
& =\left(C_{k+1}\left(P_{k}+Q\right) C_{k+1}^{H} R^{-1}\right)^{-1} \\
& \cdot\left(C_{k+1} P_{k+1} C_{k+1}^{H} R^{-1}\right) .
\end{aligned}
$$

4. At least in linear CS with a constant sensing matrix the resolution of consecutive frequency bins is also to be considered in discrete Fourier transform (cf. [40] and the references therein).

\section{References}

[1] E. P. A. M. Bakkers, M. T. Borgström, and M. A. Verheijen, "Epitaxial growth of III-V nanowires on group IV substrates," MRS Bulletin, vol. 32, no. 2, pp. 117-122, 2007.

[2] Y. B. Bolkhovityanov and O. P. Pchelyakov, "GaAs epitaxy on Si substrates: modern status of research and engineering," PhysicsUspekhi, vol. 51, no. 5, pp. 437-456, 2008.

[3] Y. Cui and C. M. Lieber, "Functional nanoscale electronic devices assembled using silicon nanowire building blocks," Science, vol. 291, no. 5505, pp. 851-853, 2001.

[4] X. Duan, Y. Huang, Y. Cui, J. Wang, and C. M. Lieber, "Indium phosphide nanowires as building blocks for nanoscale electronic and optoelectronic devices," Nature, vol. 409, pp. 6669, 2001.

[5] M. H. Huang, S. Mao, H. Feick et al., "Room-temperature ultraviolet nanowire nanolasers," Science, vol. 292, no. 5523, pp. 1897-1899, 2001.

[6] M. S. Gudiksen, L. J. Lauhon, J. Wang, D. C. Smith, and C. M. Lieber, "Growth of nanowire superlattice structures for nanoscale photonics and electronics," Nature, vol. 415, no. 6872, pp. 617-620, 2002.

[7] T. Akiyama, K. Nakamura, and T. Ito, "Effects of stacking sequence on the electrical conductivity of InAs: a combination of density functional theory and Boltzmann transport equation calculations," Japanese Journal of Applied Physics, vol. 54, no. 7, Article ID 075001, 2015.

[8] A. Biermanns, D. Carbone, S. Breuer et al., "Distribution of zinc-blende twins and wurtzite segments in GaAs nanowires probed by X-ray nanodiffraction," Physica Status Solidi-Rapid Research Letters, vol. 7, no. 10, pp. 860-863, 2013.

[9] H. Shi, B. Dong, and W. Wang, "Features of twins and stacking faults in silver nanorice and electron-beam irradiation effect," Nanoscale, vol. 4, no. 20, pp. 6389-6392, 2012.

[10] H. N. Chapman and K. A. Nugent, "Coherent lensless X-ray imaging," Nature Photonics, vol. 4, no. 12, pp. 833-839, 2010.

[11] J. W. Miao, T. Ishikawa, I. K. Robinson, and M. M. Murnane, "Beyond crystallography: diffractive imaging using coherent $\mathrm{X}$ ray light sources," Science, vol. 348, no. 6234, pp. 530-535, 2015.

[12] A. L. Patterson, "A fourier series method for the determination of the components of interatomic distances in crystals," Physical Review, vol. 46, no. 5, pp. 372-376, 1934.

[13] R. W. Gerchberg and W. O. Saxton, "Practical algorithm for determination of phase from image and diffraction plane pictures," Optik, vol. 35, no. 2, pp. 237-250, 1972.

[14] J. R. Fienup, "Reconstruction of an object from the modulus of its Fourier transform," Optics Letters, vol. 3, no. 1, pp. 27-29, 1978.

[15] J. R. Fienup, "Phase retrieval algorithms: a comparison," Applied Optics, vol. 21, no. 15, pp. 2758-2769, 1982.

[16] I. Robinson and R. Harder, "Coherent X-ray diffraction imaging of strain at the nanoscale," Nature Materials, vol. 8, no. 4, pp. 291-298, 2009.

[17] A. Davtyan, A. Biermanns, O. Loffeld, and U. Pietsch, "Determination of the stacking fault density in highly defective single GaAs nanowires by means of coherent diffraction imaging," New Journal of Physics, vol. 18, no. 6, Article ID 063021, 2016.

[18] P. M. Pelz, M. Guizar-Sicairos, P. Thibault, I. Johnson, M. Holler, and A. Menzel, "On-the-fly scans for X-ray ptychography," Applied Physics Letters, vol. 105, no. 25, Article ID 251101, 2014. 
[19] R. Horstmeyer, R. Y. Chen, X. Ou, B. Ames, J. A. Tropp, and C. Yang, "Solving ptychography with a convex relaxation," New Journal of Physics, vol. 17, no. 5, Article ID 053044, 2015.

[20] V. Favre-Nicolin, J. Eymery, R. Koester, and P. Gentile, "Coherent-diffraction imaging of single nanowires of diameter 95 nanometers," Physical Review B, vol. 79, Article ID 195401, 2009.

[21] S. S. Chen, D. L. Donoho, and M. A. Saunders, "Atomic decomposition by basis pursuit," SIAM Review, vol. 43, no. 1, pp. 129-159, 2001.

[22] E. J. Candès, Y. C. Eldar, T. Strohmer, and V. Voroninski, "Phase retrieval via matrix completion," SIAM Journal on Imaging Sciences, vol. 6, no. 1, pp. 199-225, 2013.

[23] A. Chai, M. Moscoso, and G. Papanicolaou, "Array imaging using intensity-only measurements," Tech. Rep., Stanford University, Stanford, Calif, USA, 2010.

[24] M. Fornasier, H. Rauhut, and R. Ward, "Low-rank matrix recovery via iteratively reweighted least squares minimization," SIAM Journal on Optimization, vol. 21, no. 4, pp. 1614-1640, 2011.

[25] E. J. Candès, J. K. Romberg, and T. Tao, "Stable signal recovery from incomplete and inaccurate measurements," Communications on Pure and Applied Mathematics, vol. 59, no. 8, pp. 12071223, 2006.

[26] D. L. Donoho, “Compressed sensing," IEEE Transactions on Information Theory, vol. 52, no. 4, pp. 1289-1306, 2006.

[27] S. Foucart and H. Rauhut, A Mathematical Introduction to Compressive Sensing, Birkhäuser, 2013.

[28] N. Vaswani, "Kalman filtered compressed sensing," in Proceedings of the IEEE International Conference on Image Processing (ICIP '08), pp. 893-896, San Diego, Calif, USA, October 2008.

[29] D. Kanevsky, A. Carmi, L. Horesh, P. Gurfil, B. Ramabhadran, and T. N. Sainath, "Kalman filtering for compressed sensing," in Proceedings of the 13th Conference on Information Fusion (FUSION '10), pp. 1-8, IEEE, Edinburgh, Scotland, July 2010.

[30] O. Loffeld, T. Espeter, and M. Heredia Conde, "From weighted least squares estimation to sparse CS reconstruction," in Proceedings of the IEEE 3rd International Workshop on Compressed Sensing Theory and its Applications to Radar, Sonar and Remote Sensing (CoSeRa '15), pp. 149-153, Pisa, Italy, June 2015.

[31] O. Loffeld, A. Seel, M. Heredia Conde, and L. Wang, "A nullspace based L1 minimizing Kalman filter approach to sparse CS reconstruction," in Proceedings of the 11th European Conference on Synthetic Aperture and Radar, June 2016.

[32] S. J. Julier and J. K. Uhlmann, "New extension of the Kalman filter to nonlinear systems," in Proceedings of the Signal Processing, Sensor Fusion, and Target Recognition VI, vol. 3068 of Proceedings of SPIE, Orlando, Fla, USA, April 1997.

[33] N. Correll, Introduction to Autonomous Robots, CreateSpace Independent Publishing Platform, 2014.

[34] B. E. Warren, X-Ray Diffraction, Dover, 1990.

[35] M. Barchuk, V. Holý, D. Kriegner, J. Stangl, S. Schwaiger, and F. Scholz, "Diffuse x-ray scattering from stacking faults in a-plane GaN epitaxial layers," Physical Review B-Condensed Matter and Materials Physics, vol. 84, no. 9, Article ID 094113, 2011.

[36] P. S. Maybeck, Stochastic Models, Estimation, and Control, vol. 1 of Mathematics in Science and Engineering, Academic Press, 1979.

[37] G. H. Golub and C. F. Van Loan, Matrix Computations, John Hopkins, 2013.

[38] Y. Chi, L. L. Scharf, A. Pezeshki, and A. R. Calderbank, "Sensitivity to basis mismatch in compressed sensing," IEEE
Transactions on Signal Processing, vol. 59, no. 5, pp. 2182-2195, 2011.

[39] M. F. Duarte and R. G. Baraniuk, "Spectral compressive sensing," Applied and Computational Harmonic Analysis, vol. 35, no. 1, pp. 111-129, 2013.

[40] G. Tang, B. N. Bhaskar, P. Shah, and B. Recht, "Compressed sensing off the grid," IEEE Transactions on Information Theory, vol. 59, no. 11, pp. 7465-7490, 2013.

[41] T. Valkonen, "A primal-dual hybrid gradient method for nonlinear operators with applications to MRI," Inverse Problems, vol. 30, no. 5, Article ID 055012, 2014.

[42] A. Chambolle and T. Pock, "A first-order primal-dual algorithm for convex problems with applications to imaging," Journal of Mathematical Imaging and Vision, vol. 40, no. 1, pp. 120-145, 2011.

[43] W. Hackbusch, Iterative Lösung Großer Schwachbesetzter Gleichungssysteme, Lemma 2.10.2, Teubner, 1993. 


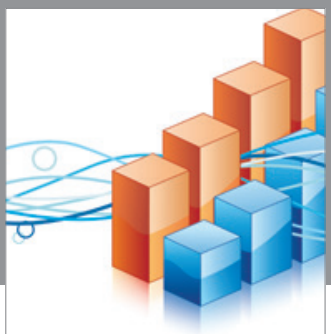

Advances in

Operations Research

vatem alat4

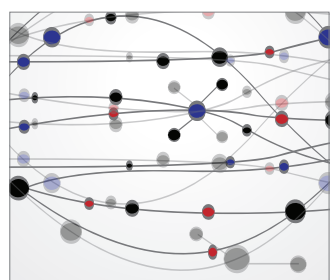

\section{The Scientific} World Journal
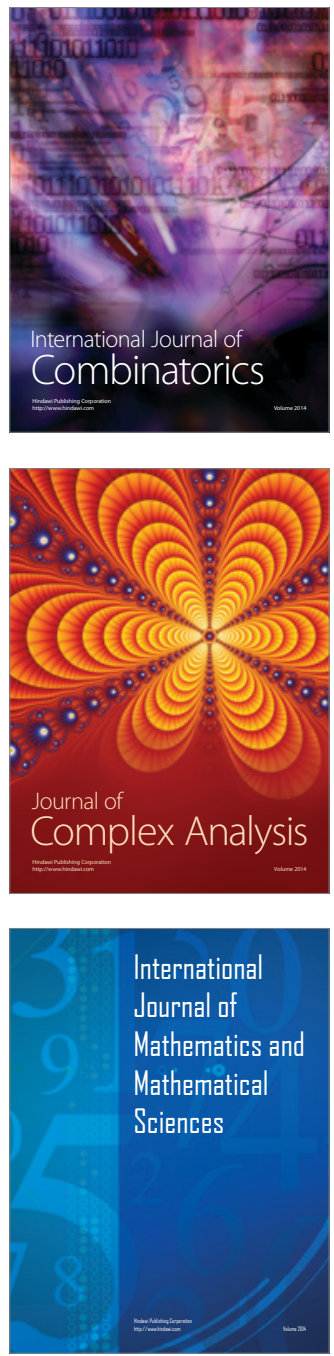
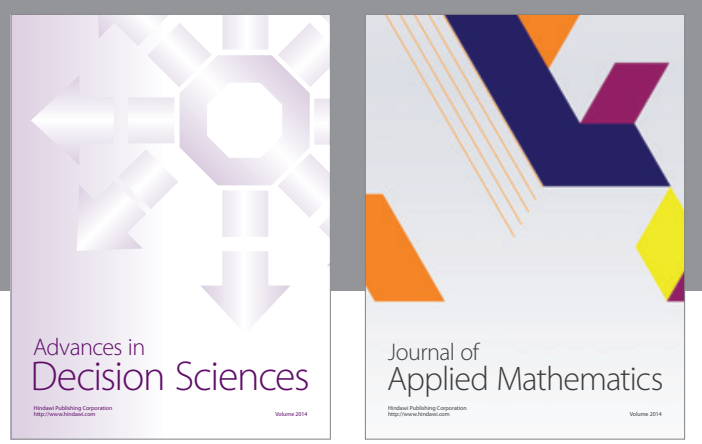

Algebra

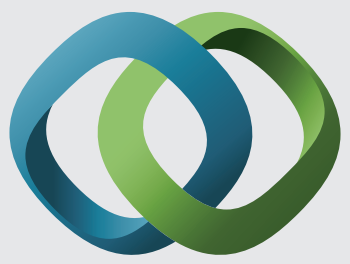

\section{Hindawi}

Submit your manuscripts at

http://www.hindawi.com
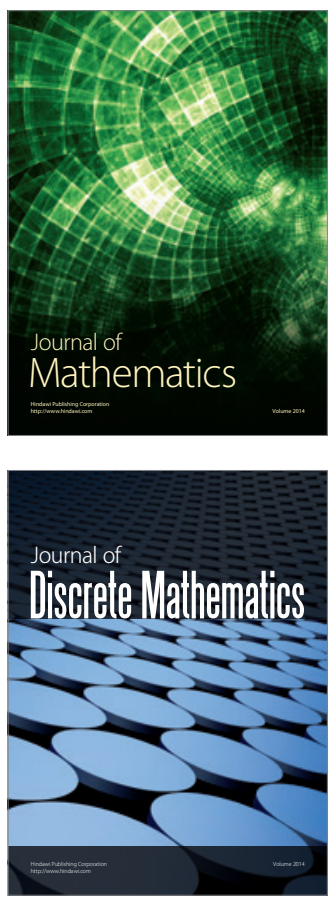

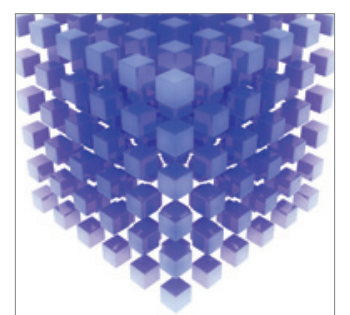

Mathematical Problems in Engineering
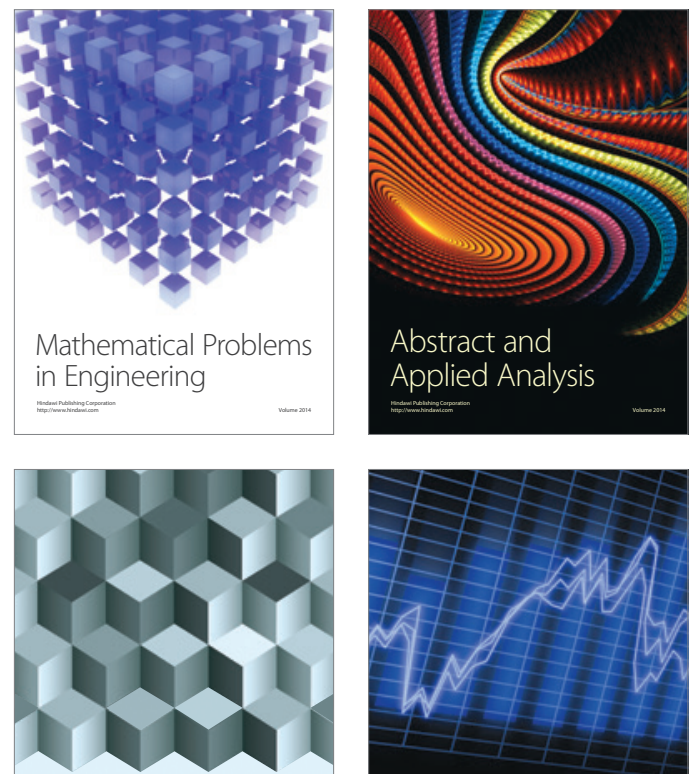

Journal of

Function Spaces

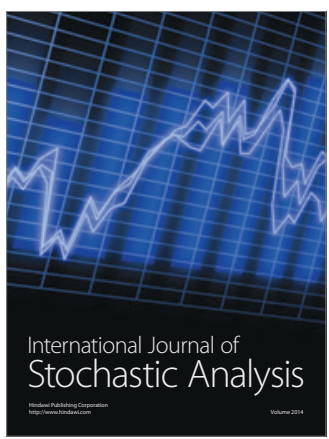

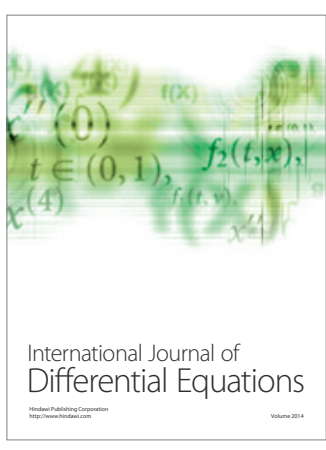
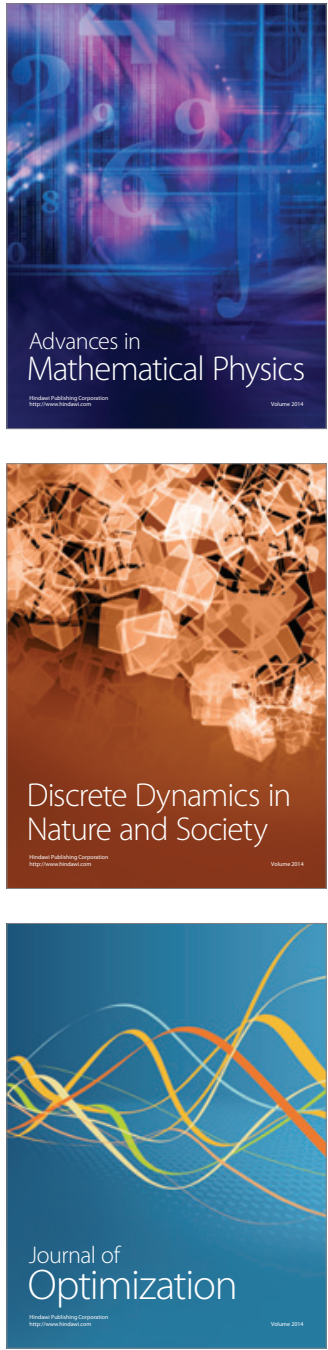